\title{
Long-term mortality in patients with ST-segment elevation myocardial infarction is associated with anti-citrullinated protein antibodies
}

Maaike P.J. Hermans, $\mathrm{MD}^{1^{\star}}$, Daniel van der Velden, MSc, $\mathrm{PhD}^{2^{\star}}$, José M. Montero Cabezas, $\mathrm{MD}^{3}$, Hein Putter, MD, PhD', Tom W.J. Huizinga, MD, PhD5, Johan Kuiper, MD, $\mathrm{PhD}^{6}$, Rene E.M. Toes, $\mathrm{PhD}^{7}$, Martin J. Schalij MD, $\mathrm{PhD}^{8}, \mathrm{~J}$. Wouter Jukema, $\mathrm{MD}, \mathrm{PhD}^{9^{*}}$, Diane van der Woude, MD, $\mathrm{PhD}^{10^{*}}$ ${ }^{*}$ Contributed equally to manuscript

1. PhD Candidate at Department of Cardiology, Leiden University Medical Center, Leiden, the Netherlands. This author takes responsibility for all aspects of the reliability and freedom from bias of the data presented and their discussed interpretation.

2. Researcher at Department of Rheumatology, Leiden University Medical Center, Leiden, the Netherlands. This author takes responsibility for all aspects of the reliability and freedom from bias of the data presented and their discussed interpretation.

3. Senior Medical Specialist at Department of Cardiology, Leiden University Medical Center, Leiden, the Netherlands. This author takes responsibility for all aspects of the reliability and freedom from bias of the data presented and their discussed interpretation.

4. Professor Department of Medical Statistics and Bioinformatics, Leiden University Medical Center, Leiden, the Netherlands. This author takes responsibility for all aspects of the reliability and freedom from bias of the data presented and their discussed interpretation.

5. Professor and Head of Department of Rheumatology, Leiden University Medical Center, Leiden, the Netherlands. This author takes responsibility for all aspects of the reliability and freedom from bias of the data presented and their discussed interpretation.

6. Professor and Head of Department of Therapeutic Immunomodulation, Faculty of Science, Leiden Academic Centre for Drug Research/BioPharmaceutics, Leiden, the Netherlands. This author takes responsibility for all aspects of the reliability and freedom from bias of the data presented and their discussed interpretation.

7. Professor and Staff Member of Department of Rheumatology, Leiden University Medical Center, Leiden, the Netherlands. This author takes responsibility for all aspects of the reliability and freedom from bias of the data presented and their discussed interpretation. 
8. Professor and Head of Department of Cardiology, Leiden University Medical Center, Leiden, the Netherlands. This author takes responsibility for all aspects of the reliability and freedom from bias of the data presented and their discussed interpretation.

9. Professor and Staff Member at Department of Cardiology, Leiden University Medical Center, Leiden, the Netherlands. This author takes responsibility for all aspects of the reliability and freedom from bias of the data presented and their discussed interpretation.

10. Senior Medical Specialist at Department of Rheumatology, Leiden University Medical Center, Leiden, the Netherlands. This author takes responsibility for all aspects of the reliability and freedom from bias of the data presented and their discussed interpretation.

Corresponding author: J. Wouter Jukema, MD, PhD

Department of Cardiology, Leiden University Medical Center

Albinusdreef 2, 2333 ZA Leiden, the Netherlands

Phone: +31 715262020 | Fax: +31 715266809 | E-mail: J.W.Jukema@lumc.nl

Acknowledgment of grant support: none

Keywords: anti-citrullinated protein antibodies; ST-segment elevation myocardial infarction 


\section{Structured Abstract}

Background: Cardiovascular (CV) mortality is higher in patients with rheumatoid arthritis (RA), in particular when anti-citrullinated protein antibodies (ACPA) are present. Recently, ACPA have also been described in a cohort of patients without RA, but with coronary artery disease (CAD). It is however unknown if ACPA can consistently be found in patients with CAD, and if ACPA are associated with mortality in these patients. The purpose of this study was to assess the relationship between ACPA and long-term outcomes including mortality in patients with ST-elevation myocardial infarction (STEMI) without RA.

Methods: All patients with STEMI from the MISSION! Intervention Study were analyzed. Patients with RA were excluded. The association between ACPA (anti-CCP3) at baseline and 10 year mortality and re-infarction was investigated.

Results: In total, 29 (11\%) of 275 included patients were ACPA-positive, substantiating the previous description of ACPA in CAD patients. Increased cumulative cardiac mortality was observed in ACPApositive patients in comparison with ACPA-negative patients. Moreover, after correction for other associated factors, ACPA-positivity was associated with long-term mortality (HR 3.1 [Cl 1.4-7.1] pValue $=0.01]$ and long-term combined endpoint of re-infarction and death (HR 2.4 [1.2-4.6] p-Value $=$ $0.01)$

Conclusion: In STEMI patients without RA, the presence of ACPA is independently associated with long-term mortality and the combined endpoint of re-infarction and death. ACPA in patients with and without RA might act as an independent pro-atherogenic factor. 


\section{Introduction}

Inflammatory processes are known to play a key role in the development of coronary artery disease (CAD). Coronary atherosclerotic lesions are characterized by macrophage activation, fibrosis, production of matrix metalloproteinases (MMPs) and lipid deposition, factors which are associated with chronic low-grade inflammation. This might be the reason that $C A D$ and vulnerable plaques are more prevalent in patients with chronic inflammatory diseases like rheumatoid arthritis (RA) (1).

In RA approximately $60 \%$ of patients harbor antibodies directed against citrullinated proteins: socalled anti-citrullinated protein antibodies (ACPA) (2). Citrulline is a post-translational modification of the amino acid arginine by the enzyme peptidylarginine deiminase (PAD), a process that frequently occurs in the context of inflammation (3). Interestingly, it has been shown that the cardiovascular risk is significantly higher in ACPA-positive versus ACPA-negative RA patients (4). Furthermore, it has been reported that citrullination of proteins also occurs in myocardium and atherosclerotic lesions $(5$, 6). Although ACPA is thought to be very specific for RA (98\%) (7), a recent study surprisingly found that $10 \%$ of the CAD patients without RA were ACPA-positive (8). Likewise, rheumatoid factor (RFhas also been reported to be associated with a high cardiovascular risk in patients without RA (ref Tomasson et al, Ann Rheum Dis 2010; 69: 1649-54).

This raises the question whether 1) ACPA can consistently be found in non-RA patients with CAD and 2) whether ACPA are associated with mortality in these patients. Therefore, we investigated if ACPA are associated with long-term mortality and re-infarction in patients without RA with established CAD. 


\section{Methods}

\section{Patients and outcomes}

The present study used data from patients with ST-segment elevation myocardial infarction (STEMI), who were included in the MISSION! Intervention Trial. The MISSION! Intervention Trial was conducted from February 2004 to October 2006 and investigated the use of either Bare Metal Stents (BMS) or Sirolimus Eluting Stents (SES) during primary percutaneous coronary intervention (PCI).(9) All patients were treated according to the institutional STEMI protocol, based on guidelines of the European Society of Cardiology, American College of Cardiology and the American Heart Association. $(10,11)$ At inclusion, data were collected regarding patient demographics, risk factors and clinical characteristics. SYNTAX score was used to assess CAD complexity by using baseline angiographic images obtained during the index procedure after reperfusion of the culprit segment (12). Calculations were performed by an experienced interventional cardiologist. Patients with prior myocardial infarction $(n=11)$ or prior revascularization $(n=7)$ were excluded. In addition, chart review was performed for all ACPA-positive patients to determine whether they had a previous rheumatic disease, or developed a rheumatic disorder during the long-term follow-up period. These patients $(n=8)$ were also excluded for analysis. To investigate long-term outcome, 10 year all-cause mortality and the combined endpoint of 10 year all-cause mortality and re-infarction (with and without STsegment elevation) was assessed. Information on all-cause mortality was obtained from the Dutch Municipality Records registry. Cause of death was retrieved from general practitioners. Follow-up data on serious adverse events including myocardial infarction, new revascularisation and stroke was obtained by telephone interviews at 2, 5 and 10 years after admission. The study was approved by the institutional ethical committee. Written informed consent was obtained from all patients before enrolment in the study.

\section{Autoantibody testing}

Baseline serum samples drawn before primary PCI were used ACPA analysis. ACPA positivity was determined using a commercially available ELISA system for anti-CCP3 testing (Quanta Lite ${ }^{\mathrm{TM}}$ CCP3.1 IgG/IgA Cat\# 704550, INOVA Diagnostics Inc., US). According to supplier's manual, values above $20 \mathrm{aU} / \mathrm{mL}$ were considered positive. ACPA-levels in patients from the MISSION Intervention 
trial were compared with ACPA-levels derived from sera of 160 healthy controls aged between 20 and 70 years from the Leiden area (13).

\section{Statistical analysis}

Normally distributed continuous variables were reported as mean and standard deviation, and compared with Student's t-test. Skewed distributed continuous variables were reported as median and interquartile range, and compared with Mann-Whitney $\mathrm{U}$ test. Categorical variables were reported as number and percentage, and compared with Pearson's chi-square test. Event-free survival was analyzed with Kaplan-Meier estimates and compared between groups with the log-rank test. Cox regression was performed to assess the association between the ACPA-positivity and primary and secondary outcome measures. Other variables used for the analysis included: sex, BMI, previous hypertension and/or smoking, out of hospital cardiac arrest, anterior infarction, infarct size, number of vessels diseased (>50\%), complete revascularization, culprit vessel, proximal lesion, TIMI flow before intervention and drug-eluting stent during primary $\mathrm{PCl}$. Variables were used in multivariable models in case the univariable $p$-value was $<0.10$. All statistical tests were performed with SPSS software (Version 22.0, SPSS IBM Corp., Armonk, New York). P-Values $<0.05$ assessed by two-sided tests were considered to be statistically significant. 


\section{Results}

A total of 275 STEMI patients from the MISSION! Intervention study had no history of RA or RA during the follow-up period and were included in the analysis. A positive ACPA test was found in 11\% (29/275) of the included patients, which is significantly more than the $2 \%(3 / 160)$ of ACPA positive tests in a healthy control population ( $p$-Value $<0.01$ ) (Figure 1). ACPA-positive and ACPA-negative patients did not differ significantly with regard to demographics, risk factors, clinical and angiographic characteristics. However, there were some differences between ACPA-positive and ACPA-negative patients that reached no statistical significance: age, female sex, infarct size, multi-vessel CAD and Creactive protein (CRP) levels (Table 1$)$.

Median long-term follow-up was 9.4 years [IQR 8.7-9.9]. In ACPA-positive patients, cumulative mortality was significantly higher than in ACPA-negative patients (ACPA-positive patients 8/29 (28\%) vs. ACPA-negative patients 27/246 (11\%), (log-rank p-Value < 0.01) (Figure 2A). Cardiac cause of death was more prevalent in ACPA-positive patients (ACPA-positive patients 4/29 (14\%) vs. ACPAnegative patients 9/246 (4\%), log-rank $p$-Value $<0.01)$, which was either heart failure or re-infarction. The combined long-term endpoint of re-infarction and/or death occurred more frequently in ACPApositive patients as well (ACPA-positive patients 11/29 (38\%) vs. ACPA-negative patients 54/246 (22\%), log-rank p-Value $=0.03)($ Figure 2B).

To assess if advanced coronary atherosclerosis might be in the causal pathway of ACPA-positivity and re-infarction and/or death, SYNTAX score after reperfusion was included in the multivariable Cox regression analysis. Even then ACPA-positivity remained independently associated with long-term mortality (HR 3.1 [95\% Cl 1.4-7.2] p-Value < 0.01) and re-infarction and/or death (HR 2.4 [95\% Cl 1.24.6] $\mathrm{p}$-Value $=0.01)($ Table 2). No significant correlation was found between ACPA levels and continuous SYNTAX scores $(p=0.392)$. 


\section{Discussion}

Key findings of this study are: 1) approximately $11 \%$ of non-RA patients with established CAD are ACPA-positive, corroborating previous results, and 2) ACPA-positivity is independently associated with long-term mortality and/or re-infarction in these patients.

ACPA are widely recognized as the most disease-specific marker for RA. Moreover, ACPA are associated with a more severe disease course in RA with e.g. more joint destruction. The most potent risk factors for RA have been shown to be specifically associated with ACPA-positive disease: genetic features (HLA, the majority of predisposing SNPS) and smoking (14). This raises the question whether these factors are also associated with the presence of ACPA in RA-negative patients. In this study, current smoking status was not significantly different between ACPA-positive and -negative CAD patients. Further studies are therefore required to elucidate whether genetic predisposition is also associated with ACPA development in CAD patients without RA.

Interestingly, we also found an association between ACPA levels and long-term clinical outcomes in patients with ACPA levels below the cutoff of $20 \mathrm{aU} / \mathrm{mL}$ (data not shown). This cut-off yields optimal sensitivity and specificity for diagnosing RA, but lower levels might well be meaningful in other settings. Our findings indicate that even low ACPA levels might play a role in the progression of CAD.

The association between ACPA and with long-term outcome in patients with CAD without RA in our study, is in line with the current evidence of the prognostic role of ACPA in patients with RA for development of either cardiovascular (CV) disease and death (Hansson et al., 2011, 14, 15). The fact that we now find an association between ACPA and CAD in the absence of RA, raises interest in the role of these autoantibodies and anti-citrulline autoimmunity in the atherosclerotic disease process. One could envisage a contribution of these autoantibodies to atherosclerosis in the following manner: citrullinated proteins have been demonstrated in both atherosclerotic plaques and perivascular interstitium $(5,6)$. If ACPA bind to these proteins, it appears likely that they would be able to exert their known effects of complement activation and the engagement of Fc-receptors on macrophages, leading to the production of inflammatory cytokines $(7,14)$. This could augment the inflammatory processes already taking place in the vessel walls in the context of atherosclerosis. Therefore the 
presence of ACPA, both in patients with and without RA, could accelerate plaque formation and rupture, and thereby lead to increased mortality (3).

The exact citrullinated antigens in the vasculature remain to be identified. In clinical studies including patients with RA, high levels of ACPA targeting specifically citrullinated histone $2 \mathrm{~B}$ were associated with subclinical atherosclerosis, whereas the association between ACPA targeting citrullinated fibrinogen and subclinical atherosclerosis could not be demonstrated $(16,17)$.

Enhancement of the inflammatory process of atherosclerosis by ACPA would explain why the rate of ACPA-positivity is significantly higher in patients with STEMI compared to healthy controls, and why these patients have a higher risk of re-infarction and/or death. We determined if advanced coronary atherosclerosis (as measured by the SYNTAX score after reperfusion) was in the causal pathway of ACPA-positivity and re-infarction and/or death by adjusting the multivariable analyses for SYNTAX-

score. However, ACPA-positivity remained significantly associated with the clinical outcomes. There are several possible explanations for this finding; either SYNTAX-score may in this case not completely capture or reflect coronary atherosclerosis, or ACPA might exert their effect via a different pathway.

The association between ACPA-positivity and progressive CAD warrants further study. If ACPA are indeed found to be pro-atherogenic, this may alter the common concept of the pathophysiology of CVD. As a consequence, immunosuppression could become a novel treatment option, as is already being investigated. (18) Additionally, if future research confirms our findings, ACPA could be a new biomarker for CVD and predict a worse long-term outcome.

\section{Limitations}

This study is the first to describe the association of long-term mortality and/or re-infarction and ACPA in patients without RA. Limitations of the current study are the observational study design, precluding conclusions about causality, and the use of 1 cohort with a limited sample size. Therefore, we were unable to test associations in subgroups, restricting the applicability of the findings to other patient groups. However, the results of our study confirm previous findings, indicating reproducibility. Strengths are the long duration of follow-up and stringent control for and exclusion of patients who developed RA. The treatment according to a strict protocol both in-hospital and during outpatient 
clinical follow-up excludes the possibility that differences in treatment account for the observed results.

\section{Conclusion}

In conclusion, we found that the presence of ACPA was independently associated with long-term mortality and/or re-infarction in patients with myocardial infarction without RA. ACPA might therefore act as an independent pro-atherogenic factor in patients both with and without RA. Future research is required to provide more insight into the development of anti-citrulline autoimmunity in patients with $C A D$ without $R A$, and into the underlying mechanism causing more progressive $C A D$ in these patients. 
Acknowledgments

None 


\section{References}

[1] Hansson GK, Hermansson A. The immune system in atherosclerosis. Nat Immunol 2011; 12:204-12.

[2] Willemze A, Trouw LA, Toes RE, Huizinga TW. The influence of ACPA status and characteristics on the course of RA. Nat Rev Rheumatol 2012; 8:144-52.

[3] Vossenaar ER, Zendman AJ, van Venrooij WJ, Pruijn GJ. PAD, a growing family of citrullinating enzymes: genes, features and involvement in disease. Bioessays 2003; 25:1106-18.

[4] Farragher TM, Goodson NJ, Naseem H, et al. Association of the HLA-DRB1 gene with premature death, particularly from cardiovascular disease, in patients with rheumatoid arthritis and inflammatory polyarthritis. Arthritis Rheum 2008; 58:359-69.

[5] Giles JT, Fert-Bober J, Park JK, et al. Myocardial citrullination in rheumatoid arthritis: a correlative histopathologic study. Arthritis Res Ther 2012; 14:R39.

[6] Sokolove J, Brennan MJ, Sharpe O, et al. Brief report: citrullination within the atherosclerotic plaque: a potential target for the anti-citrullinated protein antibody response in rheumatoid arthritis. Arthritis Rheum 2013; 65:1719-24.

[7] Suwannalai P, Trouw LA, Toes RE, Huizinga TW. Anti-citrullinated protein antibodies (ACPA) in early rheumatoid arthritis. Mod Rheumatol 2012; 22:15-20.

[8] Cambridge G, Acharya J, Cooper JA, Edwards JC, Humphries SE. Antibodies to citrullinated peptides and risk of coronary heart disease. Atherosclerosis 2013; 228:243-6.

[9] van der Hoeven BL, Liem SS, Dijkstra J, et al. Stent malapposition after sirolimus-eluting and bare-metal stent implantation in patients with ST-segment elevation myocardial infarction: acute and 9-month intravascular ultrasound results of the MISSION! intervention study. JACC Cardiovasc Interv 2008; 1:192-201.

[10] O'Gara PT, Kushner FG, Ascheim DD, et al. 2013 ACCF/AHA guideline for the management of ST-elevation myocardial infarction: a report of the American College of Cardiology Foundation/American Heart Association Task Force on Practice Guidelines. Circulation 2013; 127:e362-425.

[11] Steg PG, James SK, Atar D, et al. ESC Guidelines for the management of acute myocardial infarction in patients presenting with ST-segment elevation. Eur Heart J 2012; 33:2569-619.

[12] Sianos G, Morel MA, Kappetein AP, et al. The SYNTAX Score: an angiographic tool grading the complexity of coronary artery disease. Eurolntervention 2005; 1:219-27.

[13] Magro-Checa C, Schaarenburg RA, Beaart HJ, Huizinga TW, Steup-Beekman GM, Trouw LA. Complement levels and anti-C1q autoantibodies in patients with neuropsychiatric systemic lupus erythematosus. Lupus 2016; 25:878-88.

[14] Clavel C, Nogueira L, Laurent L, et al. Induction of macrophage secretion of tumor necrosis factor alpha through Fcgamma receptor Ila engagement by rheumatoid arthritis-specific autoantibodies to citrullinated proteins complexed with fibrinogen. Arthritis Rheum 2008; 58:678-88. 
[15] Ajeganova S, Humphreys JH, Verheul MK, et al. Anticitrullinated protein antibodies and rheumatoid factor are associated with increased mortality but with different causes of death in patients with rheumatoid arthritis: a longitudinal study in three European cohorts. Ann Rheum Dis 2016.

[16] Montes A, Corrales A, Calaza M, et al. Brief report: lack of replication of an association between anti-citrullinated fibrinogen and subclinical atherosclerosis in patients with rheumatoid arthritis. Arthritis Rheumatol 2015; 67:2861-5.

[17] Geraldino-Pardilla L, Giles JT, Sokolove J, et al. Association of Anti-Citrullinated Peptide Antibodies with Coronary Artery Calcification in Rheumatoid Arthritis. Arthritis Care Res (Hoboken) 2016.

[18] Cardiovascular Risk Reduction Study (Reduction in Recurrent Major CV Disease Events) (CANTOS) [Internet]. ClinicalTrials.gov [Internet]. 2011. 


\section{Figure legends}

Figure 1. ACPA levels in the MISSION! Intervention cohort $(n=275)$ and a healthy control group $(n=$ 160). The horizontal line indicates the upper limit of the normal range of $20 \mathrm{aU} / \mathrm{mL}$, considered CCP3.1 positive.

Figure 2. A) Long-term cumulative mortality in ACPA-positive vs. ACPA-negative patients. B) Longterm cumulative re-infarction/mortality in ACPA-positive vs. ACPA-negative patients. 


\section{Tables}

Table 1. Baseline characteristics of ACPA-positive and ACPA-negative patients with STEMI.

\begin{tabular}{|c|c|c|c|c|}
\hline & & $\begin{array}{c}\text { ACPA-negative } \\
\text { (ACPA } \leq 20 \mathrm{aU} / \mathrm{mL} \text { ) }\end{array}$ & $\begin{array}{c}\text { ACPA-positive } \\
\text { (ACPA>20 aU/mL) }\end{array}$ & \\
\hline & & $N=246$ & $N=29$ & \\
\hline & & no. (\%) & no. (\%) & $\mathrm{p}$-Value \\
\hline Age, mean (SD), y & & $58.4(11.6)$ & $62.7(10.4)$ & 0.06 \\
\hline Woman & & $55(22)$ & $10(34)$ & 0.15 \\
\hline Body mass index, mean (SD), kg/m² & & $26.5(4.1)$ & $27.0(5.5)$ & 0.59 \\
\hline Treated hypertension $\dagger$ & & $70(28)$ & $6(21)$ & 0.38 \\
\hline Diabetes & & $23(9)$ & $2(7)$ & 0.66 \\
\hline Treated hyperlipidemiał & & $44(18)$ & $4(14)$ & 0.58 \\
\hline Current smoker & & $136(55)$ & $16(55)$ & 0.99 \\
\hline Family history of CVD & & $108(44)$ & $14(48)$ & 0.67 \\
\hline Out of hospital cardiac arrest & & $7(3)$ & $0(0)$ & 0.36 \\
\hline Cardiogenic shock & & $5(2)$ & $0(0)$ & 0.45 \\
\hline Anterior infarction & & $128(55)$ & $17(63)$ & 0.41 \\
\hline Infarct size, median (IQR), g/m² & & $8.6(4.4-15.4)$ & $13.1(4.7-18.7)$ & 0.25 \\
\hline \multirow[t]{3}{*}{ Number of vessels diseased (>50\%) } & 1 & $141(58)$ & $13(45)$ & 0.18 \\
\hline & 2 & $92(38)$ & $15(52)$ & 0.14 \\
\hline & 3 & $11(5)$ & $1(3)$ & 0.79 \\
\hline Complete revascularization & & $244(100)$ & $29(100)$ & $\sim$ \\
\hline \multirow[t]{3}{*}{ Culprit vessel } & $\mathrm{RCA}$ & $74(30)$ & $8(28)$ & 0.78 \\
\hline & LAD & $133(54)$ & $17(59)$ & 0.64 \\
\hline & LCX & $39(16)$ & $4(14)$ & 0.77 \\
\hline Proximal lesion & & $126(51)$ & $12(41)$ & 0.32 \\
\hline TIMI 0 flow before intervention & & 169 (69) & $20(71)$ & 0.81 \\
\hline Drug-eluting stent & & $124(50)$ & $11(38)$ & 0.20 \\
\hline
\end{tabular}


Reversible ischemia post-infarct

myoview

Abbreviations: ACPA, anti-citrullinated protein antibodies; BMI, body mass index; CABG, coronary artery bypass graft surgery; CVD, cardiovascular disease; IQR, Interquartile Range; PCI, percutaneous coronary intervention; SD, standard deviation † Defined as systolic blood pressure $\geq 140 \mathrm{~mm} \mathrm{Hg}$ and/or diastolic blood pressure $\geq 90 \mathrm{~mm} \mathrm{Hg}$ and/or the use of antihypertensive medication.

‡ Serum total cholesterol $\geq 6.0 \mathrm{mmol} / \mathrm{L}$ and/or serum $\mathrm{TG} \geq 2.2 \mathrm{mmol} / \mathrm{L}$ or treatment with lipid lowering drugs. 
Table 2. Univariable and multivariable Cox regression analysis for 10-years all-cause mortality and 10-years re-infarction or death.

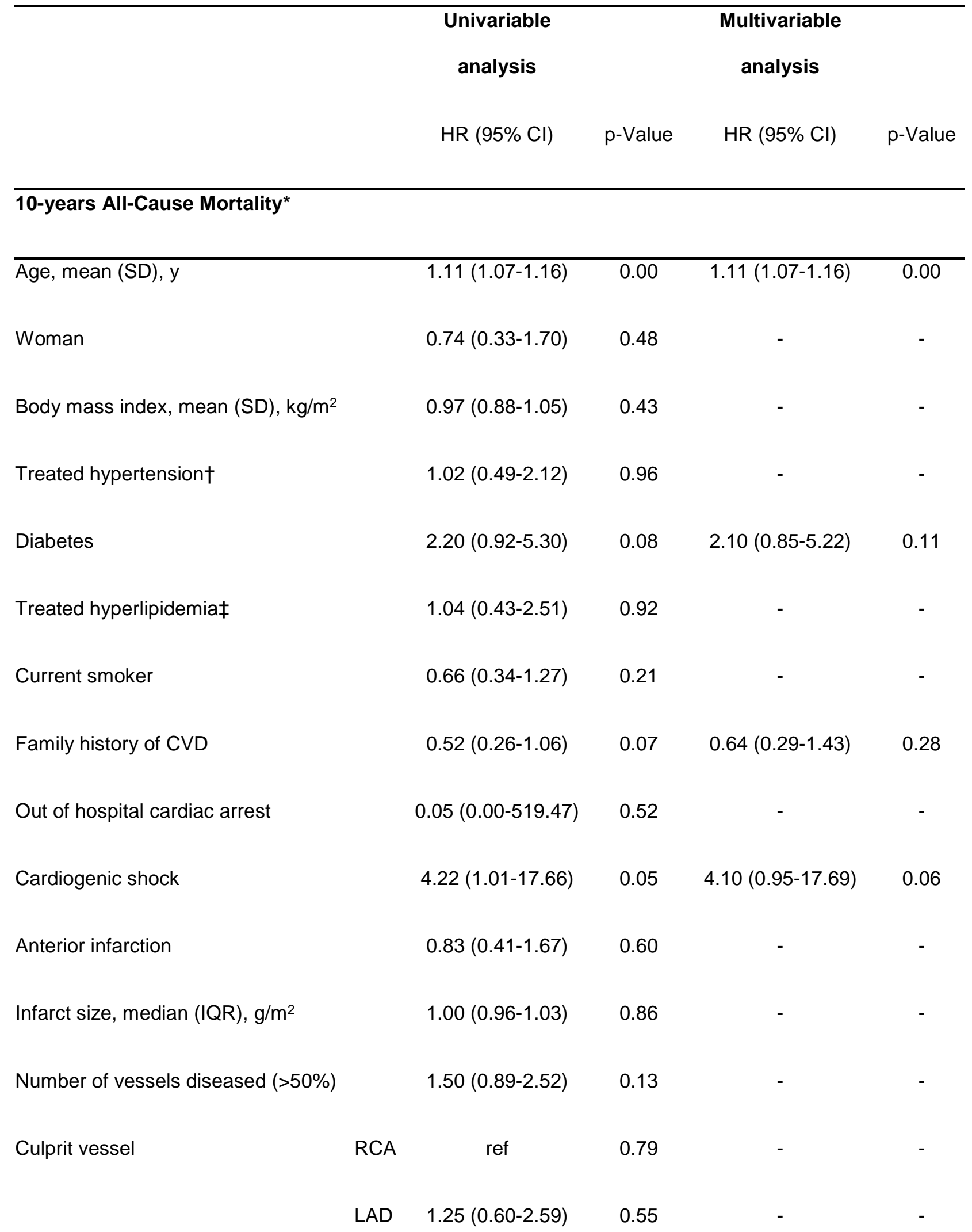




$$
\text { LCX } \quad 1.27(0.51-3.21) \quad 0.61
$$

Proximal lesion

TIMI 0 flow before intervention

Drug-eluting stent

Reversible ischemia post-infarct

myoview

SYNTAX score after reperfusion

ACPA $>20 \mathrm{aU} / \mathrm{mL}$

10-years Re-infarction or Death*
$0.54(0.24-1.23) \quad 0.14$

$0.99(0.94-1.04) \quad 0.66$

$3.05(1.39-6.73) \quad 0.01$

$3.12(1.36-7.16) \quad 0.01$

\begin{tabular}{|c|c|c|c|c|}
\hline Age, mean (SD), y & $1.02(1.00-1.04)$ & 0.07 & $1.02(0.99-1.04)$ & 0.18 \\
\hline Woman & $0.79(0.44-1.42)$ & 0.43 & - & - \\
\hline Body mass index, mean (SD), $\mathrm{kg} / \mathrm{m}^{2}$ & $1.00(0.95-1.06)$ & 0.91 & - & - \\
\hline Treated hypertension $\dagger$ & $0.89(0.52-1.55)$ & 0.69 & - & - \\
\hline Diabetes & $2.09(1.07-4.10)$ & 0.03 & $2.00(0.96-4.14)$ & 0.06 \\
\hline Treated hyperlipidemiał & $1.92(1.11-3.32)$ & 0.02 & $1.90(1.04-3.49)$ & 0.04 \\
\hline Current smoker & $1.07(0.66-1.72)$ & 0.80 & - & - \\
\hline Family history of CVD & $0.62(0.38-1.02)$ & 0.06 & $0.52(0.30-0.91)$ & 0.02 \\
\hline Out of hospital cardiac arrest & $0.05(0.00-32.00)$ & 0.36 & - & - \\
\hline Cardiogenic shock & $1.73(0.42-7.15)$ & 0.45 & - & - \\
\hline Anterior infarction & $0.82(0.50-1.35)$ & 0.43 & - & - \\
\hline
\end{tabular}


Infarct size, median (IQR), g/m²

Number of vessels diseased (>50\%)

Culprit vessel

Proximal lesion

TIMI 0 flow before intervention

Drug-eluting stent

Reversible ischemia post-infarct

myoview

SYNTAX score after reperfusion

ACPA $>20 \mathrm{aU} / \mathrm{mL}$
$1.00(0.97-1.03)$

0.95

$1.31(0.88-1.94)$

0.18

RCA

ref

0.71

LAD

$1.23(0.72-2.10)$

0.45

\section{LCX $\quad 1.22(0.62-2.40) \quad 0.57$}

$1.36(0.84-2.20) \quad 0.21$

$0.83(0.50-1.39) \quad 0.48$

$1.13(0.70-1.82) \quad 0.62$
$2.10(0.96-4.61) \quad 0.07$

$2.07(1.08-3.95) \quad 0.03$
$2.32(1.04-5.15)$

0.04

$2.35(1.21-4.56)$

Abbreviations: ACPA, anti-citrullinated protein antibodies; BMI, body mass index; CABG, coronary artery bypass graft surgery; CVD, cardiovascular disease; $\mathrm{PCl}$, percutaneous coronary intervention ${ }^{\star}$ Causes of death were:

in ACPA-negative patients: COPD (2), CVA (1), heart failure (3), infection (2), malignancy (10), myocardial infarction (4), sudden cardiac death (2), unknown (3);

in ACPA-positive patients: Heart failure (1), liver failure (1), malignancy (2), myocardial infarction (2), sudden cardiac death (1), unknown (1).

† Defined as systolic blood pressure $\geq 140 \mathrm{~mm} \mathrm{Hg}$ and/or diastolic blood pressure $\geq 90 \mathrm{~mm} \mathrm{Hg}$ and/or the use of antihypertensive medication.

‡ Serum total cholesterol $\geq 6.0 \mathrm{mmol} / \mathrm{L}$ and/or serum $\mathrm{TG} \geq 2.2 \mathrm{mmol} / \mathrm{L}$ or treatment with lipid lowering drugs. 